\title{
Opportunities and perspectives of PMU application in power districts with distributed energy resources
}

\author{
Pavel Ilyshin ${ }^{1 *}$, Alexey Mokeev ${ }^{2}$, and Vladimir Narovlyanskii ${ }^{3}$ \\ ${ }^{1}$ Petersburg power engineering institute of professional development, Aviation str. 23, Saint-Petersburg, Russia \\ ${ }^{2}$ Northern (Arctic) Federal University, Severnaya Dvina emb.17, Arkhangelsk, Russia \\ ${ }^{3}$ Energosetproject, Tkatskaya str. 1, Moscow, Russia
}

\begin{abstract}
The report examines the application of PMUs for various functional purposes of identifying emergency conditions, implement functions of relay protection and automation devices, perform a number of monitoring and diagnostic functions of the equipment. The analysis of functional requirements for control systems, protection and automation of power districts with distributed energy resources is carried out. The description of certain algorithms of emergency control and mode control is presented. Promising fields of scientific research are identified to expand the range of functions that can be implemented with the use of PMU.
\end{abstract}

\section{Introduction}

The development of modern telecommunication technologies and phasor management units (PMUs) enables development of distributed monitoring, protection and management systems WAMPACS [1, 2]. With PMUs emerging on the market, a device with small mass-size indicators and cost comparable with the cost of multifunction measuring transducers, the scope of the new units expands significantly [3]. One of the promising areas of PMU application is power districts with distributed energy resources (DER).

The main advantages of the PMU are related to the possibility of calculating parameters of power-district mode on the basis of current and voltage synchrophasors according to the fundamental harmonic at any control level, including the active values of the currents and voltages, active, reactive and full powers, symmetric components parameters mode etc. Based on six current and voltage synchrophasors, about one hundred parameters for the main harmonic can be calculated for each connection, and with the help of current and voltage synchrophasors obtained from various connections of the power district, the parameters of the power district in question can be calculated. This opens up wide opportunities for managing normal and emergency conditions both in power districts with distributed energy resources and in power systems to which these power districts are connected.

\section{Prospects and issues of DER development}

Main trends in DER development are as follows:
Increase in gross installed power of various types of DER in the energy systems of developed countries, - Increase in the installed power of individual DER units,

- Harmonious development of conventional generation and distributed energy resources.

As for primary equipment of power plants and power grid facilities, the key areas of development are determined by energy saving trends, development of distributed generation with a wide range of various drive motors and independent generation in large industrial enterprises using non-traditional and renewable energy resources.

An important structural feature of power supply networks of industrial enterprises with independent generation is small values of mutual resistances of generators and loads. This leads to a significant mutual influence of the operating modes of the generating units on the load operation modes and vice versa [4].

The main challenges in the implementation of the DER are conditioned by following factors:

Positive and negative voltage deviations in all nodes of the network due to the mutual influence of power consumption schedules and power generation by DER facilities,

Presence of reversible power flows in low and medium voltage networks,

The added complexity of relay protection diagrams in low and medium voltage networks and the principles of applied protection and automation devices,

Increase in the level of Isc in low and mediumvoltage networks,

The need to ensure sustainability of the power system when a large number of generating units of the DER facilities are disconnected, 
The need to ensure the possibility of isolated operation of all types of generating units at the DER facilities

To effectively solve the problems mentioned above, it is necessary to introduce new technologies in control systems, relay protection and automation in power districts with distributed energy resources. Let us first consider the requirements for modern control, protection and automation systems in power districts with DER.

\section{The functionality of control, protection and automation systems}

The following main functions of modern automated process control systems, relay protection devices and automation of power districts with DER are identified:

Ensuring observability and controllability of electrical modes,

Efficient control over the choice of the composition of the operating generating equipment,

Provision of group control over active power and voltage taking into account regulatory ranges and technological limitations,

Implementation of the function of automatic frequency control and active power flows in an isolated power district,

Implementation of automatic allocation for a balanced load in the event of damage to the supply network or the mode of high risks of a power failure,

Automatic turn of generator sets (GS)/power plant from zero from cold and hot conditions,

Automatic provision of specified network consumption parameters/power delivery to the network of the GS/power plant,

Detection and prevention of the emergence of asynchronous modes at the GS and the links of the power district with the power system,

Control of electromechanical transient processes in the power district at the rate of the process along a given trajectory,

Application of high-speed devices of relay protection generators, transformers, busbars,

The use of centralized earth-fault protection,

Monitoring of the operation of DER equipment with control over the current mode and generation of emergency warnings,

Removal of static and dynamic load characteristics of various technological productions,

Calculation of technical and economic indicators.

Most of the functions listed above can be most effectively implemented using phasor management unit technology. At the same time, the functional capabilities of control, protection and automation systems using PMUs are significantly wider at the same financial costs than when applying traditional methods of building control, protection and automation systems.

\section{Phasor management units}

Until recently, the main deterrents for the widespread use of PMUs in distribution networks and power districts with DER were associated with the high cost of these devices and the large mass-size indicators of PMU. Therefore, the scope of application was mainly limited to the wide-area management systems (WAMS).

The experience of implementing the PMU in the WAMS and the progress in microelectronics provided the development of devices with small mass-size dimensions and low cost comparable to the cost of multifunction telemetry measurement transducers. This enables forecasting mass application of such devices at substations of various voltage classes, including 6-20 kV substations and those in power districts with DER.

At the same time, the scope of PMUs is significantly expanded: process management automated systems, automated process control systems, mode and emergency control automatics, incl. most advanced control, protection and automation systems WAMPACS. Therefore, in this article, the PMUs term refers not only to devices used in the WAMS, but also the systems listed above.

When used as part of automated control systems, devices with synchronized phasor measurements (SPM) support require measurements of the network mode parameters taking into account higher harmonics, and it can be implemented by measuring equivalent current and voltage synchrophasors [6]. This allows calculating about a hundred parameters of the network mode considering the highest harmonics at any level of control.

Expansion of the PMUs scope of application is associated with the expansion of the functionality of these devices for a number of applications, primarily for use in the system of relay protection and emergency automatics, where additional requirements for the quality of processing of input signals are imposed on the PMUs. When using synchronized vector measurements for emergency control and relay protection devices, it is important to ensure the reliability of measurements of synchrophasors in conditions of electromagnetic transients. At the same time, devices supporting SPM should provide suppression of free components of electromagnetic transients and research in this field has been actively conducted in recent years [5]. When using synchrophasors in protection and automation devices, an additional increase in the rate of data transmission is required.

For the application of the SPM technology as part of the relay protection and emergency control systems, the measurement ranges of the current synchrophasors corresponding to the emergency modes need to be extended. Similar devices are developed by both foreign and Russian manufacturers [3].

In addition, in the creation of modern control, protection and automation systems, it is expedient to integrate SPM technologies into the structure of a digital substation. Experience in implementing certain digital substation technologies, especially fast GOOSE messages, PTP time synchronization protocol and HSR and PRP backup protocols, have proved their high efficiency in substation and power plant automation systems. Taking into account the accumulated experience, as well as continuous improvement of 
microcontrollers and signal processors, implementation of these technologies does not have a significant effect on the final cost of devices anymore.

Current and voltage synchrophasors can be used as an alternative to sampling values which in fact represent raw digitized values of currents and voltages. When using synchrophasors instead of selective values of current and voltage, the traffic on the process bus decreases drastically, implementation of longitudinal differential protections and differential bus protectors, centralized control devices for normal and emergency operation modes is simplified.

The primary advantage of using synchrophasors for implementation of emergency control automation is the high quality of measurements of the network mode parameters on the basis of current and voltage synchrophasors for electromechanical transient processes, which is especially important in the implementation of the UFLS, under-frequency islanding system, OSPS and a number of other emergency control systems. It is also promising to use synchrophasors in the implementation of mode automation, for example, providing centralized control of reactive power compensation and maintaining the voltage in load nodes.

These data pave the way to the emergence of inexpensive and compact PMU on the market for use in distribution networks and in power districts with DER for the purpose of performing control, protection and automation functions.

Currently, the PMUs is used for simultaneous measurement of current and voltage synchrophasors. But in most practical cases, it is advisable to use separate PMUs systems for current (from the measuring and relay winding of the current measuring transformers) and for voltage (from the star and the open triangle windings of the measuring voltage transformers).

The main advantages of such an implementation of the PMUs, along with the reduction of cost and overall dimensions of these devices, are related to the need to avoid multiple duplication of voltage sync measurements in the devices of each bay or bus system taking into accountthe rare application of combined current and voltage measuring transformers in projects.

Experience in the implementation of the PTP time synchronization protocol in a number of domestic intelligent devices in the structure of a digital substation has proved its effectiveness for the PMUs. There is no need for a separate synchronization cable, as opposed to the case of time synchronization using PPS or IRIG-A/B.

In the coming years, we should expect a massive application of PMUs for solving management, protection and automation issues. But even a small number of PMU can improve the assessment of the power system condition [2, 7]. The same is true for the possibilities of implementing certain tasks of emergency control.

Consider the effectiveness of the application of PMU in the example of the implementation of a complex device for pole slip protection (OSPS) for a power facility.

\section{OSPS of a power facility}

OSPS of a power facility for integrated protection against the asynchronous mode (AM) of the power facility as a whole and its parts. Here, the term "power facility" means a local electric power complex which may include generation, substations, local load, power lines, distribution network feeders and other elements.

The technical solution for the complex protection of the power facility is based on the use of a distributed system of PMU connected with the central analytical unit by fibre-optic data transmission lines.

OSPS of the power facility provides protection:

When passing the AM section along the lines of communication of the facility with the backbone network (OSPS network function),

When passing the AM section of transformers and other power elements of the power plant scheme (OSPS of the power facility circuits-function),

If there is an asynchronous move of one of the generators of the power plant (Generator OSPS).

Operation of OSPS is considered using the functional diagram shown in Fig. 1, on the example of a conventional power plant with a substation through which power is delivered to the network.

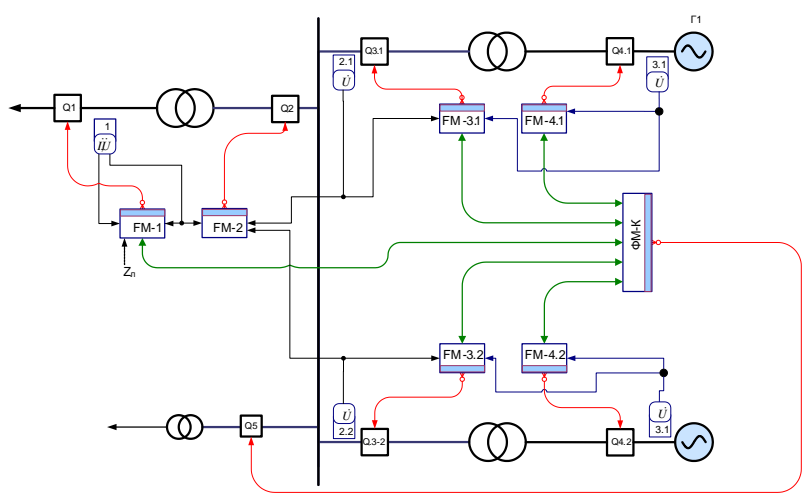

Fig. 1. Example of the OSPS functional diagram.

When implementing the network OSPS, the voltage and current synchrophasors of the PMU-1 are used, as well as the setting for the resistance of the monitored network section (function module FM-1). Operation of this OSPS is the same as that of the ALAR-M device [810]. At the same time, OSPS can be implemented both as OSPS by resistance and angular OSPS.

A more advanced version of OSPS is related to the implementation of the minimum stress points (MSP) detecting method [9]. To determine the MSP, it is necessary to know only the synchronized stress vectors in the nodes of the power district with DER. The method allows increasing the efficiency and completeness of AM detection without the need for preliminary calculation of OSPS settings, to anticipate weak links and thereby to increase the reliability of analysis and maintenance of the mode, reduce the number of failures, excessive and false alarms of emergency control devices. Fig. 2 is a diagram of control zones for possible sections in asynchronous mode. 
The functional module FM-1 monitors the power system - power plant zone. If AM occurs, the section of which passes along the lines of the power output of the power plant, the power plant is separated from the power system by issuing a signal for the B1 shutdown.

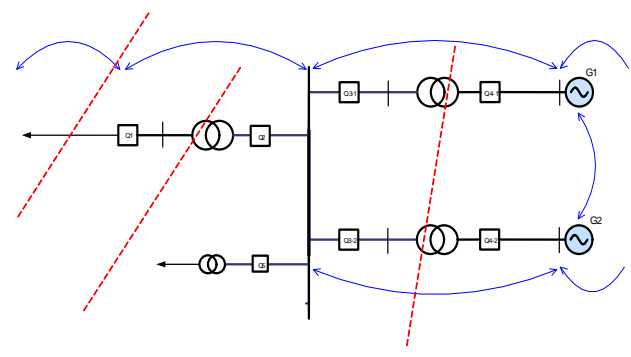

Fig. 2. Control zones of asynchronous mode sections.

OSPS in power plant circuits uses syncrovector stress measurements from PMU-1 PMU-2.1 and PMU-2.2 installed on the sides of the controlled circuit and outputs a signal of the occurrence of AM when detecting a predetermined value of the difference angle (often as a crank of $180^{\circ}$ ). The functional module FM-2 monitors the zone of network transformers of the power plant. If AM occurs, the section of which passes through transformers of the power plant, the power plant is separated from the power system by issuing a signal to turn off B1 and in some cases to turn off B2.

OSPS in generator circuits functions similarly to OSPS in power plant circuits, but it is connected to the transformer generator busbar trunking measuring voltage circuits and is implemented via functional modules FM3.1 and FM-3.2. This functionality does not require prior settings.

If AM occurs, the section of which passes through the power circuits of the generator, the generator is separated from the power plant by issuing a signal to turn off the breaker B3 (B3.1 in the circuits of generator $1, \mathrm{~B} 3.2$ in the circuits of generator 2) and in some cases to turn off circuit breaker B4 (B4 .1 in the circuits of the generator 1; B4.2 in the circuits of the generator 2).

OSPS of the generator is implemented by means of the functional module FM-4 and uses data from PMU-3 and information from the generator shaft run sensor. FM-4 monitors development of the asynchronous run of the generator, including accidents in the excitation system of the generator. If an asynchronous run occurs, the generator is separated from the power plant by issuing a signal to turn off B4 (B4.1 in the circuits of generator 1, B4.2 in the circuits of generator 2).

Functional module FM-K provides coordinated operation of FM-1-FM-5 modules and implements:

Separation of the power plant from the power system when the section of AM passes through the power-circuit zones of all the generators of the power plant, i.e. when the accident is not due to technical failures of the generators' operation but is caused by the AM occurrence in the network,

Operation of the OSPS generator functional unit by using the comparison of the angles of the voltage vectors on the power plant buses with the voltage vector angle on the stator of the generator in the absence of a generator rotation sensor or in addition to it,

Monitoring of mutual angles of power plant generators for monitoring (and damping) of oscillations, identifying sources of low-frequency oscillations (LFO) and preventing the development of AM of generators due to tightening of stable generators in the oscillatory process.

Detection of the relative «abnormal» motion (oscillations and/or asynchronous motion) of one of the generators relative to other generators of the power plant is made by mutual comparison of the angles between the vectors of the direct sequence voltages on the stators of the generators or the values of the rotor angles of the generators.

Application of OSPS based on PMU will allow monitoring of mutual oscillation of power plant generators. The oscillation of the generator of the power plant in relation to other generators notably occurs due to inaccurate adjustment of the excitation and speed regulators, provoking the occurrence of the LFO and thereby contributing to a reduction in the stability margin.

Insignificant fluctuations of synchronous generators of power plants occur almost always due to continuous unbalances of the consumed and generated capacities. These oscillations are easily damped and do not cause complications, but in the event of significant changes in the mode, cyclic changes in frequency with amplitudes around $0.05 \mathrm{~Hz}$ may occur.

In this case, the behaviour of each generator (generator-turbine unit) of the plant may differ from the behaviour of adjacent generators due to differences in electromechanical characteristics (inertia, intrinsic resonance frequencies, etc.), as well as the settings of the excitation and speed regulators. This particularly applies to power plants where generators with different values of mechanical inertia constants or generators of different types and brands are installed.

In such a situation, it is important to directly monitor the behaviour of generators, and it will provide the basis for developing recommendations for tuning the excitation and speed regulators in order to reduce the mutual oscillation of the rotors of the power plant generators.

Monitoring generator oscillations allow assessing the risk of development of asynchronous move, the risk of tightening adjacent generators in the process of oscillating, it also allows ensuring generation and output of commands to the emergency control system of generators.

The frequency of formation of the voltage and current synchrophasors should not exceed $20 \mathrm{~ms}$ (basic value). It is useful to provide increased frequency of vector formation with a periodic sequence of 1 to $10 \mathrm{~ms}$ to ensure reliable operation of OSPS at the plants with low-inertia generators.

\section{Conclusions}

At present, there is a tendency to increase the number of 
industrial power districts with distributed energy resources in the form of distributed generation facilities and various energy storage devices in which mutual influence of the operation modes of generating units and load is high.

The increase management efficiency in power districts with distributed resources of energy is largely determined by the quality of data coming from control objects.

The use of PMUs for various functional purposes can effectively enable detection of hazardous modes, implementation of functions of relay protection and automation devices, the performance of a number of functions for monitoring and diagnosing equipment in power districts with DER.

The use of PMUs allows implementation of both a centralized function of the OSPS power plant at a novel technological level and decentralized and combined one depending on the tasks assigned.

The functional capabilities of control, protection and automation systems using PMUs are significantly higher than when traditional ways of building these systems are applied, with the financial costs being equal for both options.

\section{References}

1. Wide area protection \& Control technologies. CIGRE, Working Group B5.14 (Paris, 2016).

2. M. Kezunovic, S. Meliopoulos, V. Venkatasubramanian, V. Vittal. Application of Time-Synchronized Measurements in Power System Transmission Networks (Springer, NY, 2014).

3. A. Mokeev, Digital Substation. URL: http://digitalsubstation.com.

4. P. Ilyshin, Energoexpert, 1, 58-62 (2015).

5. A. Mokeev, Proceedings IEEE International Conference SIBCON (2017).

6. A. Mokeev, V. Bovykin, A. Miklashevich, D. Ulyanov. Proceedings International Conference Actual trends in development of Power System Relay Protection and Automation (2015).

7. A. Gamm, A. Glazunova, Yu. Grishin, I. Kolosok, E. Korkina, Electrical Technology Russia, 6, 2-9 (2009).

8. P. Ilyshin, P. Chusovitin, Relay Protection and Automation, 4, 16-22 (2014).

9. V. Narovlyanskii, V. Kurmak, Power Plant, 3, 48-51 (2012).

10. V. Narovlyanskii, Modern Methods and Means of Prevention of Asynchronous Operation of the Power Grid (Energoatomizdat, Moscow, 2004). 